\title{
AN INSIGHT ON THE WEAKENING OF THE INTERLAYER BONDS IN A CAMEROONIAN KAOLINITE THROUGH DMSO INTERCALATION
}

\author{
MBEY J. A. ${ }^{1,2 *}$, THOMAS F. ${ }^{1}$, NGALLY SABOUANG C. J. ${ }^{2}$, LIBOUM $^{2}$ and NJOPWOUO \\ D. ${ }^{2}$
}

1 Laboratoire Environnement et Minéralurgie, UMR 7569 CNRS-INPL, 15 Avenue du Charmois, B.P. 40. F-54501, Vandoeuvre-lès-Nancy Cedex 2 Laboratoire de Physico-chimie des Matériaux Minéraux, Département de Chimie Inorganique, Université de Yaoundé I, B.P. 812 Yaoundé

MBEY Jean Aimé: jean-aime.mbey@univ-lorraine.fr

NGALLY SABOUANG Cyrill Joël: cngally@yahoo.fr

LIBOUM: $\underline{\text { liboum2002@yahoo.fr }}$

NJOPWOUO Daniel: dnjop@yahoo.fr

THOMAS Fabien: fabien.thomas@ univ-lorraine.fr

* Corresponding author: e-mail: jean-aime.mbey@univ-lorraine.fr or mbey25@yahoo.fr;

Tel: +23799238925

This is the authors version of the article published in Applied Clay Science:

An insight on the weakening of the interlayer bonds in a Cameroonian kaolinite through DMSO intercalation

J.A. Mbey, F. Thomas, C.J. Ngally Sabouang, D. Njopwouo Applied Clay Science 83, 327-335.

http://www.sciencedirect.com/science/article/pii/S0169131713002378 
24 ABSTRACT : In this study, intercalation of dimethylsulphoxide (DMSO) in a cameroonian kaolinite is used to achieve weakening of the interlayer hydrogen bonds, in the perspective of dispersion or even exfoliation of the clay within polymer composite materials. Displacement of intercalated DMSO by ethyl acetate and ammonium acetate is studied in order to simulate the interactions with the polymer matrix. The exfoliation of the kaolinite is well evidenced by X-ray diffraction and SEM observations. The disruption of the interlayer bonds is shown by the displacement of the FT-IR vibration modes of both Al-OH and Si-O functions, and by the decrease of the dehydroxylation temperature recorded by Controlled Rate Thermal Analysis. Complete displacement of DMSO by ethyl acetate is achieved and the crystalline structure is deeply disordered as a result of interlayer bonds weakening. The displacement of DMSO by ammonium acetate leads to a ternary composite of DMSO/ammonium acetate with respective intercalation ratio of $62.4 \%$ and $57.7 \%$.

Key words: Kaolinite, Intercalation, Weakening, Interlayer bond, Composite.

\section{INTRODUCTION}

Clays are growingly used in the industry as mineral fillers in polymers composite materials, due to their high aspect ratio and high specific area that determine the intensity of the claypolymer interactions (Luo and Daniel, 2003). It has been demonstrated for more than two decades that many properties of polymer materials, such as the mechanical or thermal properties, or water and gas barrier effect, can be improved by incorporation of clay particles in the polymer (Arora and Padua, 2010; Pavlidou and Papaspyrides 2008; Ray and Bousmina, 2005; Alexandre and Dubois, 2000).The key factor to achieve such improvements is the dispersion of the clay particles within the polymer matrix. Therefore montmorillonite is most commonly used in polymer-clay composites because of its high surface area and expansible nature of its crystallites which allows complete delamination in aqueous medium (Cabedo et al., 2004).

Kaolinite is widely used in the paper industry as a glossy surface agent in coated papers, diluting agent of titanium dioxide, white pigment, paint extender, or rubber filler (Murray, 
2000; Conceicao et al., 2005). Conversely, its use in polymer-clay composites is by far less common (de Carvalho et al., 2001; Whilhem et al., 2003; Chen and Evans, 2005). However, kaolinite is the most ubiquitous clay. Natural kaolinite deposits are sometimes of such purity level that there is not much additional purification required prior to industrial use, since only little contamination with illite/muscovite, quartz, rutile, ilmenite, or feldspar is observed (Murray, 1988). In Africa, the occurrence of kaolinite clay was recently evaluated, and it appears that the exploitation of the occurrences is still to be improved, which offers both investment and research opportunities (Ekosse, 2010). The use of kaolinite as reinforcing filler in the production of polymer-clay (nano)composite is one of the potential applications of interest, and represents the general aim of the present work.

The asymmetrical structure due to the superposition of the tetrahedral and the octahedral sheet in the kaolinite layer induces strong superposed dipoles, which, in conjunction with hydrogen bonds between the silicone oxide ring and the aluminol surface result in strong cohesive energy of the mineral (Cabedo, 2004; Giese, 1988). In addition, the crystalline network of kaolinite is practically devoid of isomorphic substitutions, and does not require charge compensation of hydrated interlayer cations. These characteristics cause kaolinite to occur as non-expandable, large particles of low anisotropy, which explains the lack of interest up to now for its use as mineral filler in polymer-clay composites.

For a convenient use of kaolinite in exfoliated state, one must use a route that ensures weakening of the interlayer bonding between the kaolinite layers, prior to its dispersion within a polymer matrix. There are few organic molecules that can be directly intercalated within the kaolinite. This is the case for dimethylsulfoxide (DMSO), N-methylformamide, acetamide, formamide, potassium acetate; ammonium acetate (Frost et al., 2010; Frost et al., 2003; Itagaki et al., 2001; Frost et al., 1999; Olejnik et al., 1970; Olejnik et al., 1968). These 
molecules are divided into three types: (i) compounds such as urea or formamide which contain two distinct groups to accept and donate hydrogen; (ii) compounds with a high dipole moment, such as dimethyl-sulfoxyde (DMSO); (iii) ammonium, potassium, rubidium and caesium salts of short-chain fatty acids (acetate, propionate) (Lagaly et al., 2006; Oleijnik et al., 1970). The displacement of the guest molecule from an intercalated kaolinite is often used to promote intercalation of other molecules (Letaief et Detellier, 2007; Cabedo et al., 2004; Itagaki et al., 2001; Komori et al., 1999). Also, the rate at which the displacement takes place is determined by the weakening of the interlayer interactions in the clay. Another interesting factor to consider is the time lasting of the intercalated molecule which, to the knowledge of the authors, was not yet evaluated in the literature, although it is evident that this factor may be of interest in the prospect of the production of a readily usable intermediate product. This paper aims at evaluating the benefit of DMSO intercalation as a pathway toward dispersion of a cameroonian kaolinite to be used within a polymer matrix for composite materials preparation. The rationale is that initial disordering of the kaolinite will determine the dispersion of the particles within the polymer matrix. For this purpose the displacement of the intercalated DMSO by the polymer matrix was simulated in low polarity and ionic medium, respectively ethyl acetate and ammonium acetate, since the polarity of the medium may have consequences on the displacement rate and hence influence the clay structure.

X Ray diffraction, infrared spectroscopy and thermal behaviour of the intercalated and raw kaolinite is used as a tool to evaluate the weakening of the layer-layer interactions and stability of the intercalated product. 


\section{MATERIAL AND METHODS}

A kaolinite from the Mayouom deposit located at the bottom of a mylonitic cliff in western Cameroon is used in this study. The genesis of this kaolin has been established by Njoya et al., (2006) as a result of a hydrothermal process. This clay has been thoroughly described in the frame of its possible use in ceramic products (Njiomou Djangang et al., 2011; Njoya et al., 2010; Nkoumbou et al., 2009; Djangang et al., 2008; Kamseu et al., 2007).

The sample used in the present study was taken at $3 \mathrm{~m}$ depth. The fraction $<40 \mu \mathrm{m}$, labelled $\mathrm{K} 3$, was collected by means of wet sieving. Using major elemental composition of the sample obtained by inductive coupled plasma by atomic emission spectrometry (ICP-AES), an approximation of the structural formula of the kaolinite phase was found to be $\left(\mathrm{Al}_{1.94}\right.$ $\left.\mathrm{Fe}_{0.06}\right)\left(\mathrm{Si}_{1.98} \quad \mathrm{Fe}_{0.02}\right) \mathrm{O}_{5}(\mathrm{OH})_{4}\left(\mathrm{Mg}_{0.02} \mathrm{Ca}_{0.002}\right)$. The mineralogical composition of the clay sample, estimated from the chemical composition (table I), the structural formula of the kaolinite phase and from the mineral composition determined by powder $\mathrm{X}$ ray diffraction (figure 1), is as follows: Kaolinite 83.3\%; Illite $10.4 \%$; Titanium oxide $3.4 \%$.

DMSO intercalated kaolinite (labelled K3-D) was prepared using the method described by Gardolinski et al. (2000), excepted that the preparation was performed at room temperature and lasted for 20 days, instead of short stirring at $60{ }^{\circ} \mathrm{C}$ followed by a lasting period of 10 days. Typically, $9 \mathrm{~g}$ of kaolinite were mixed with $60 \mathrm{~mL}$ of DMSO and $5.5 \mathrm{~mL}$ of demineralised water and left for a 20 days stay. The time-lasting of DMSO intercalation in the clay was tested after 3-years conservation of the dried intercalated samples.

Displacement of the DMSO from the kaolinite was performed using ethyl acetate and ammonium acetate by Prolabo. The respective products are labelled K3-EA and K3-AA. For the K3-AA product, $1 \mathrm{~g}$ of $\mathrm{K} 3-\mathrm{D}$ was left to stay at room temperature for 72 hours in $20 \mathrm{~mL}$ of a saturated aqueous solution of ammonium acetate. For the K3-AE product, $2 \mathrm{~g}$ of K3-D 
121 were heated under stirring at $60{ }^{\circ} \mathrm{C}$ in $15 \mathrm{~mL}$ of ethyl acetate during 5 minutes and left for a

12248 hours stay. For both preparations, the collected clay cake after gravimetric filtration on a 8

$123 \mu \mathrm{m}$ filter (Whatman grade 40) was left to dry at ambient temperature.

124 X-Ray powder diffraction patterns were recorded using a D8 Advance Bruker diffractometer

125 equipped with a Co $\mathrm{K} \alpha$ radiation $(\lambda=1.7890 \AA)$ operating at $35 \mathrm{kV}$ and $45 \mathrm{~mA}$. The

126 diffraction patterns were obtained from $1.5^{\circ}$ to $32^{\circ}$ at a scanning rate of $1^{\circ} \mathrm{min}^{-1}$.

127 Infrared spectra were recorded in diffuse reflection mode using a Bruker IFS 55 spectrometer.

128 The spectra, recorded from $4000 \mathrm{~cm}^{-1}$ to $600 \mathrm{~cm}^{-1}$ with a resolution of $4 \mathrm{~cm}^{-1}$, are 129 accumulation of 200 scans.

130 Scanning Electron Microscopy (SEM) was performed on a Hitachi S-4800 using a YAG

131 (Yttrium Aluminium Garnet) backscatter secondary electron detector for morphological

132 observation on the raw and DMSO-intercalated kaolinite.

133 Thermal analysis was performed on a home made Control Rate Thermal Analysis (CRTA)

134 apparatus equipped with a mass spectrometer. In CRTA, the temperature increase is not

135 imposed but in controlled by the reaction rate through pressure measurement. The sample is

136 placed under dynamic vacuum through a micro leak that is calibrated so that the emitted gases

137 can be quantified. The limiting pressure is fixed at $2 \mathrm{~Pa}$, which ensures a linear weight loss

138 with time. The emitted gases are analysed by a Balzers (QMS, QMA and QME 200 Pfeiffer

139 Vacuum) mass spectrometer (Feylessoufi et al., 1996).

140

141 3. RESULTS AND DISCUSSION

142 3.1. Intercalation and displacement of the DMSO molecules

143 The X-ray diffraction patterns (figure 1a) show a complete shift of the characteristic $\mathrm{d}_{001}$ peak

144 of kaolinite from $7.19 \AA$ to $11.26 \AA$. This shift is in accordance with a full monolayer 
145 intercalation of DMSO giving rise to a $4.06 \AA$ increase of the basal spacing (Fang et al., 146 2005).

147 The presence of interlayer DMSO is evidenced on the FT-IR spectrum (figure $2 \mathrm{a}$ ) by the $\mathrm{S}=\mathrm{O}$ 148 stretching at $1095 \mathrm{~cm}^{-1}$ and by the modification of the external inner surface $\mathrm{OH}$ stretching 149 mode at 3693,3668 and $3651 \mathrm{~cm}^{-1}$ (figure $2 \mathrm{~b}$ ) due to interactions of the sulfonyl group in

150 DMSO with the surface Al-OH groups of the clay. The decrease of the band at $3693 \mathrm{~cm}^{-1}$ is 151 related to the interaction of the external inner surface hydroxyl with the sulfonyl group in the 152 DMSO molecule. The bands at $3540 \mathrm{~cm}^{-1}$ and $3498 \mathrm{~cm}^{-1}$ account for the hydrogen bond of the 153 DMSO molecules to some inner surface hydroxyl group of the kaolinite layer (Johnston et al., 154 1984; Olejnik et al., 1968). The C-S-C symmetric and asymmetric stretching at $659 \mathrm{~cm}^{-1}$ and $155700 \mathrm{~cm}^{-1}$ (figure 2a) in the pure liquid DMSO are observable in the intercalated kaolinite at $156661 \mathrm{~cm}^{-1}$ and $719 \mathrm{~cm}^{-1}$ (figure 2c).

157 The bending mode of the $\mathrm{Al}-\mathrm{OH}$ (figure $2 \mathrm{~d}$ ) is shifted to higher wavelength, due to 158 interaction with DMSO. The Si-O, in plane vibrations at 1020 and $1112 \mathrm{~cm}^{-1}$ (figure 2e) are 159 shifted to lower wavelengths. These shifts are evidences for the inner surface oxygen atom 160 interactions with DMSO. Given that, interlayer bonds in kaolinite are due to $\mathrm{Al}-\mathrm{OH}$ and $\mathrm{Si}-\mathrm{O}$ 161 interactions, the interactions of these functions with DMSO, necessarily results in weakening 162 of the layer-layer interactions and therefore of the crystallite cohesion.

163 The weakening of the inter-layer interactions is further shown by the dehydroxylation 164 temperature recorded by CRTA (figure $3 \mathrm{a}$ and $3 \mathrm{~b}$ ). The dehydroxylation of the kaolinite is 165 completed at $408^{\circ} \mathrm{C}$ for the raw kaolinite, and at $275^{\circ} \mathrm{C}$ for the DMSO-intercalated kaolinite. 166 The changes in pressure are used to detect the dehydroxylation temperature. Mass 167 spectrometry measurements (figure 4a) support this observation. The ion current due to water 168 fragments is plotted as a function of time. The trend of this curves reveal three temperatures 
169 of water release (figure 4a) assigned respectively to hydration water $\left(64-90{ }^{\circ} \mathrm{C}\right)$ and to 170 structural water of the kaolinite $\left(408{ }^{\circ} \mathrm{C}\right.$ in the raw kaolinite and $273{ }^{\circ} \mathrm{C}$ in the DMSO171 intercalated kaolinite). The degradation of the intercalated DMSO is observed at $186{ }^{\circ} \mathrm{C}$ and 172 the ion currents of both the $\mathrm{S}=\mathrm{O}$ fragment $(\mathrm{m} / \mathrm{z}=48)$ and the $\mathrm{CH}_{3}-\mathrm{S}-\mathrm{CH}_{3}$ fragment $(\mathrm{m} / \mathrm{z}=$ 173 62) are consistent (figure $4 \mathrm{a}$ ) with the degradation of DMSO at $186{ }^{\circ} \mathrm{C}$. The lowering of the 174 dehydroxylation temperature is consistent with lower energy for the interlayer bonds within 175 the clay, in accordance with the conclusion drawn from FT-IR spectra, (figure $2 \mathrm{~d}$, and $2 \mathrm{~g}$ ).

176 The time lasting of the DMSO intercalation in kaolinite was clearly shown by X-ray pattern 177 as well as FTIR spectroscopy that remain unchanged after three years conservation of the 178 sample in a polyethylene bag under ambient temperature. The presented figure 3 and 4 from 179 CRTA as well as the SEM images (figure 6) were obtained using a three years old sample. 180 The comparison with a one month age sample (figure 3b), further confirms the time lasting of 181 DMSO intercalation within the kaolinite. For the one month old sample, the dehydroxylation 182 of the kaolinite is observed at $240{ }^{\circ} \mathrm{C}$. The difference between the dehydroxylation 183 temperatures for the one month and the three year old samples is possibly due to an increase 184 stability of the DMSO-Kaolinite interactions with time as a consequence of the attendant 185 release of co-intercalated water molecules.

186 Contact with ethyl-acetate leads to almost complete displacement of the DMSO as shown by 187 the disappearance of the peak at $11.26 \AA$ from XRD (figure 1b), which is characteristic of 188 DMSO intercalation. This complete displacement is also evidenced by the $\mathrm{O}-\mathrm{H}$ stretching 189 modes (figure $5 \mathrm{~b}$ ), which are almost the same for $\mathrm{K} 3-\mathrm{AE}$ and the raw $\mathrm{K} 3$, and by the absence 190 of the $\mathrm{S}=\mathrm{O}$ stretching due to DMSO at $1099 \mathrm{~cm}^{-1}$ (figure 5c). The FT IR spectrum for the K3191 AE product does not show the carbonyl vibration mode (figure 5e) and the Al-OH bending 192 returns to $916 \mathrm{~cm}^{-1}$ as observed in the raw kaolinite (figure 5f). All these observations 
193 indicate that the ethyl acetate is not intercalated. However, the stretching and bending bands

194 of $\mathrm{C}-\mathrm{H}$ on the FTIR spectrum of K3-AE (figure 5a), indicate traces of remaining DMSO

195 within the kaolinite and this presence is evidenced by mass spectroscopy during CRTA

196 (figure 4c) through the ion current of the fragments $\mathrm{m} / \mathrm{z}=48$ (for $\mathrm{S}=\mathrm{O}$ ) and $\mathrm{m} / \mathrm{z}=62$ (for

$\left.197 \mathrm{CH}_{3}-\mathrm{S}-\mathrm{CH}_{3}\right)$. The fact that ethyl acetate does not replace DMSO is probably due to the low

198 polarity of this molecule (dipole moment 1.88) because high dipole moment is a requirement

199 for intercalation in kaolinite (Lagaly et al., 2006). Hence hydrogen bonds of interest are not

200 formed between this compound and the hydroxyl groups within the kaolinite.

201 The use of ammonium acetate also leads to DMSO displacement as shown by the presence of

202 the characteristic basal peak of kaolinite at $7.15 \AA$ (figure 1b). Unlike ethyl acetate, 203 ammonium acetate partially replaces DMSO between the clay sheets. The partial 204 displacement of DMSO is evidenced by X-ray patterns, since the peak at $11.26 \AA$ due to 205 DMSO intercalation is still observable (figure $1 \mathrm{~b}$ ). Also, the IR vibrations mode of $\mathrm{S}=\mathrm{O}$ from 206 DMSO at $1095 \mathrm{~cm}^{-1}$ are detected on the IR spectrum (Figure 5c). The $14.38 \AA$ XRD peak, 207 although weak, is consistent with that described by Itagaki et al. (2001) and Sugahara et al. 208 (1988) for ammonium acetate intercalated kaolinite. It is then suggested that intercalation of 209 ammonium acetate occur in K3-AA. The FT-IR spectra clearly show the presence of 210 ammonium ions within the clay through the bending mode of $\mathrm{NH}_{4}{ }^{+}$that are observed at 1419 $211 \mathrm{~cm}^{-1}$ (figure 5b) and the carbonyl stretching mode at $1598 \mathrm{~cm}^{-1}$ (Figure 5e). The replacement 212 of DMSO by ammonium acetate is associated to the formation of interactions of greater 213 energy than DMSO interactions with the kaolinite hydroxyl groups and this is well confirmed 214 by the bending modes of $\mathrm{Al}-\mathrm{OH}$ on figure $5 \mathrm{f}$, where the $\mathrm{Al}-\mathrm{OH}$ bending mode are moved to 215 higher energy compared to the displacement due to DMSO intercalation. 
216 The ion current due to water release during CRTA (figure 4b), clearly shows that the K3-AE 217 and K3-AA have lower dehydroxylation temperatures as proof of the weakening of interlayer 218 bonds. On figure $4 \mathrm{~b}$, three dehydroxylation temperatures $\left(90^{\circ} \mathrm{C}, 362{ }^{\circ} \mathrm{C}, 417{ }^{\circ} \mathrm{C}\right)$ for $\mathrm{K} 3$-AA 219 and two $\left(90{ }^{\circ} \mathrm{C}\right.$ and $\left.392{ }^{\circ} \mathrm{C}\right)$ for $\mathrm{K} 3$-AE are observed. The dehydroxylation at $362{ }^{\circ} \mathrm{C}$ is 220 associated to the part of kaolinite co-intercalated with ammonium acetate and DMSO and at $221417{ }^{\circ} \mathrm{C}$ we have the dehydroxylation of the recovered kaolinite due to DMSO displacement. 222 The evidence for the dehydroxylation of kaolinite co-intercalated with ammonium acetate and DMSO, is given by the ion current of the fragment of $\mathrm{m} / \mathrm{z}=14$ (related to nitrogen in ammonium acetate) and the fragment of $\mathrm{m} / \mathrm{z}=48$ (related to $\mathrm{S}=\mathrm{O}$ from DMSO) (figure $4 \mathrm{~b}$ ). At $392{ }^{\circ} \mathrm{C}$ the dehydroxylation of the kaolinite recovered from the displacement of DMSO by ethyl acetate is observed and at $90{ }^{\circ} \mathrm{C}$ for both $\mathrm{K} 3-\mathrm{AA}$ and $\mathrm{K} 3-\mathrm{AE}$, the release of the hydration water is observed. The thermal stability of the kaolinite is decreased by the intercalation. Gabor et al (1995) reported the same conclusion in their study of Hydrazine and potassium acetate intercalated kaolinite. The thermal stability, of the kaolinite phase obtain after DMSO displacement, is significantly different and this is due to the induced disordering upon DMSO displacement.

232 Because the dehydroxylation of the recovered kaolinite after DMSO displacement with 233 acetate ammonium is higher for the recovered kaolinite using ethyl acetate for DMSO 234 displacement, then, one can conclude that, the kaolinite recovered by DMSO displacement 235 with ammonium acetate is less disordered. This observation suggest a high disordering by 236 displacement with ethyl acetate in comparison to displacement by ammonium acetate. The 237 displacement rate may be the cause of this difference. The thermal treatment in the synthesis 238 protocol for the $\mathrm{K} 3-\mathrm{AE}$ is probably the cause of this high displacement rate. 
The DMSO intercalation does not modify the initial kaolinite structure, since the number of

241 layers per crystallite, determined from the coherent domain thickness after the Scherrer 242 equation, remains constant (table II). The SEM micrographs (figure 6) show the 243 morphological evolution due to intercalation. On this image, one can clearly observe that clay 244 stacks appear to be thinner in the intercalated kaolinite than in the raw clay. The intercalation 245 of the kaolinite is evidenced and the conservation of the layer ordering is also observable.

246 The subsequent displacement of DMSO, strongly affects the kaolinite crystalline structure as 247 shown by the broadening and almost vanishing of the $\mathrm{d}_{001}$ peaks on the X-ray patterns (figure 248 1b). The displacement using ethyl acetate dramatically disorders the kaolinite crystallites. 249 Heller-Kallai and al. (1991) also reported crystallinity reduction in kaolinite samples after DMSO displacement by heating or washing in water. In this study, the drastic change in the crystallinity is probably due to the displacement rate which induce an important disordering 252 particularly for the K3-AE product.

253 The calculated thickness of the coherent domain in K3-EA shows an increase compared to the 254 pristine kaolinite (from $169 \AA$ to $451 \AA$ ) (table II) given a number of layers per crystallite 255 twice as much as in the raw kaolinite (from 25 to 62). In addition, the basal distance for the $256 \mathrm{~d}_{001}$ peak is substantially different from that of the pristine kaolinite $(7.29 \AA$ in K3-EA against $7.19 \AA$ in the raw material). The explanation of these differences is that high disordering 258 takes place which result in a value for the coherent domain thickness which is not associated 259 to the number of layer per crystallite but rather evidenced the random orientation of clay sheet 260 upon DMSO displacement. Consequently, the number of sheets per crystallite calculated is 261 aberrant. The displacement of DMSO using ethyl acetate does not allow the clay sheets to fall 262 down following a path close or identical to the DMSO intercalation path. In the case of 
ammonium acetate, the return path of the clay sheets is almost identical to the initial route of DMSO intercalation. The number of layer in kaolinite pseudo-crystal after DMSO displacement is 25 layers per crystallite (against 24 in the raw kaolinite) and the basal distance is $7.15 \AA$ ( 7.19 in the raw kaolinite). All of which indicates that the kaolinite phase recovered after the DMSO displacement is structurally very close to initial kaolinite. The dehydroxylation temperature from CRTA also supports this conclusion. The ammonium acetate intercalation gives rise to a ternary composite and makes it evident that the DMSO displacement is a route for composite preparation. The intercalation ratios (or 271 degree of reaction) within this ternary composite was calculated using the equation below. 272 One must keeps in mind that this equation does not consider the influence of lorentz and 273 polarization factors, the interstratification and layer shape (distortion for example). Hence the 274 relationship is used, assuming, for both expanded and unexpanded phase, the same degree of 275 particles orientation (Lagaly et al., 2006 ;Wang et Zhao, 2006; Olejnik et al.,1968).

$$
I . R=I_{\text {O01intercalate }} /\left(I_{\text {O01intercalate }}+I_{\text {O01 Kaolinite }}\right)
$$

where $I_{\text {Oolintercalate }}$ is the $\mathrm{d}_{001}$ peak intensity due to intercalation; $I_{\text {O0IKaolinite }}$ is the residual intensity of kaolinite basal peak in the intercalated product and I.R is the intercalation 279 ratio.

280 The calculated intercalation ratios are $57.7 \%$ and $62.4 \%$ respectively for ammonium acetate 281 and DMSO intercalation. The preparation conditions probably determines the intercalation 282 ratios and one can imagine that setting the preparation conditions, for DMSO displacement, 283 will help designing the desired composite. In addition, the structural differences in the 284 recovered kaolinite after DMSO displacement are due to the displacement rate. Ethyl acetate 285 leads to a rapid displacement of DMSO which contributed to higher disordering in the 
recovered kaolinite whereas ammonium acetate displacement is less rapid and allows the sheets to almost return to their initial position. This conclusion is corroborated by the results from CRTA. Hence depending on the purpose, the use of DMSO displacement as a route for composite preparation may either need a rapid or low rate for the displacement of the 290 intercalated molecule. In particular, for clay dispersion in a polymer matrix, a rapid and 291 efficient displacement may be needed.

292

293

\section{CONCLUSION}

This study shows the weakening effect of DMSO intercalation in kaolinite and point out the benefits that could be derived from DMSO displacement as a pathway for composite making. The evidence of the intercalation is clearly observed on X-ray patterns and FT-IR spectra. The interlayer interactions are weakened, as revealed by the IR bending modes of $\mathrm{Al}-\mathrm{OH}$ and the in plane vibrations of $\mathrm{Si}-\mathrm{O}$ in the kaolinite. This is further confirmed by the dehydroxylation temperature observed in CRTA. The DMSO intercalation is a time lasting modification of the kaolinite. The total displacement of the intercalated DMSO is achieved in hot ethyl acetate medium leading to a random orientation of the clay sheets in the recovered kaolinite due to a great disturbance of the clay sheets interactions. The disordered kaolinite obtained is a consequence of a rapid displacement of the intercalated DMSO. Displacement of DMSO by ammonium actetate follows a path which is similar to that of DMSO intercalation, and a ternatry composite, ammmonium acetate/DMSO/kaolinite is formed.

Hence, if conevenient and adapted conditions are set, the displacement of DMSO from DMSO intercalated kaolinite could be used as a route for composite preparation. In particular, for polymer-clay composite, a rapid displacement may help to obtain optimal dispersion and distribution of the kaolinite as filler within the polymer matrix. The present conclusions have served as basis for the preparation of cassava starch-kaolinite composite films. The 
310 evaluation of the films properties changes confirms that the use of DMSO-intercalated 311 kaolinite is advantageous and the results are subject of a companion paper (Mbey et al., 312 2012).

313 


\section{Acknowledgement}

315 University of Yaounde I (Cameroon) for laboratory facilities for the synthesis of the kaolinite 316 derivatives.

317 INPL Nancy (France) for a Doctorate research grant. 


\section{References}

319 Alexandre M. and Dubois P. (2000). Polymer-layered silicate nanocomposites: preparation, 320 properties and uses of a new class of materials. Materials Science and Engineering R, 28 ,132163

322 Arora A. and Padua G.W. (2010). Review: nanocomposites in food packaging. Journal of 323 Food Science, 75 (1), R43-R49.

324 Cabedo L., Giménez E., Lagaron J. M., Gavara R. and Saura J. J. (2004). Development of 325 EVOH-kaolinite nanocomposites. Polymer, 45, 5233-5238.

326 Chen B. and Evans J. R. G. (2005). Thermoplastic starch-clay nanocomposites and their 327 characteristics, Carbohydrate Polymers, 61, 455- 463.

328 Conceição S., Santos N. F., Velhoc J. and Ferreira J.M.F. (2005). Properties of paper coated 329 with kaolin: The influence of the rheological modifier. Applied Clay Science, 30, 165- 173

330 De Carvalho A. J. F., Curvelo A. A. S. and Agnelli J. A. M., (2001). A first insight on 331 composites of thermoplastic starch and Kaolin. Carbohydrate Polymers. 45, 189-194.

332 Djangang C. N., Elimbi A., Melo U. C, Lecomte G. L., Nkoumbou C., Soro J., Yvon J, 333 Blanchart P. and Njopwouo D. (2008). Refractory ceramics from clays of Mayouom and 334 Mvan in Cameroon . Applied Clay Science, 39, 10-18.

335 Ekosse G-I. (2010). Kaolin deposits and occurrences in Africa: Geology, mineralogy and 336 utilization. Applied Clay Science, 50, 212-236.

337 Fang Q., Huang S., and Wang W. (2005). Intercalation of dimethyl sulphoxide in kaolinite: 338 Molecular dynamics simulation study. Chemical Physics Letters, 411, 233-237.

339 Feylessoufi A., Villiérias F., Michot L. J., De Donato P., Case J.M. and Richard P. (1996). 340 Water Environment and Nanostructural Network in a Reactive Powder Concrete. Cement 341 and Concrete Composite, 18, 23-29. 
342 Frost R. L., Kristóf J., Paroz G. N. and Kloprogge J. T.(1999). Intercalation of kaolinite with 343 acetamide. Physics and Chemistry of Minerals, 26, pp 257-263.

344 Frost R. L., Makó E., Kristóf J., Horváth E. and Cseh T. (2003). The effect of 345 machanochemical activation upon the intercalation of a high-defect kaolinite with 346 formamide. Journal of colloid Interface Science, 265, 386-395.

347 Frost R. L., Kristof J. and Horvath E. (2010). Vibrational spectroscopy of intercalated 348 kaolinites. Part I. Applied Spectroscopy Reviews, 45(2),130-147.

349 Gábor M., Tóth M., Kristóf J. and Komáromi-Hiller G. (1995). Thermal and decomposition of $350 \quad$ intercalated kaolinite. Clays and Clay minerals, 43 (2), 223-228.

351 Gardolinski J. E., Carrera L. C. M. and Wypych F. (2000). Layered polymer-kaolinite 352 nanocomposites. Journal of Materials Science, 35, 3113-3119.

353 Giese R. F. (1988). Kaolin Minerals: Structures and Stabilities. In Hydrous Phyllosilicates, pp 354 29-66, ed. S.W. Bailey, Mineralogical Society of America.

355 Heller-Kallai L., Huard E. and Prost R. (1991). Disorder Induced by de-intercalation of 356 DMSO from kaolinite. Clays Minerals, 26, 245-253.

357 Itagaki T., Komori Y., Sugahara Y. and Kuroda K. (2001). Synthesis of a kaolinite-poly( $\beta$ 358 alanine) intercalation compound. Journal of Materials Chemistry, 11, 3291 - 3295.

359 Johnston C. T., Sposito G. Bocian D. F. and Birge R. R. (1984). Vibrational spectroscopic 360 study of the interlamellar kaolinite-dimethyl sulfoxide complex. Journal of Physical 361 Chemistry, 88 (24), 5959-5964.

362 Kamseu E., Leonelli C., Boccaccini D. N., Veronesi P., Miselli P., Giancarlo Pellacani , and 363 Chinje Melo U. (2007). Characterisation of porcelain compositions using two china clays 364 from Cameroon. Ceramics International, 33, 851-857. 
Komori Y., Sugahara Y. and Kuroda K. (1999). Direct intercalation of poly(vinylpyrrolidone) into kaolinite by a refined guest displacement method. Chemistry of Materials, 11, 3-6. of Clay Science, 1, 309-377, Edited by F. Bergaya, B. K.G Theng and G. Lagaly.

Letaief S. and Detellier C. (2007). Nanohybrid materials from the intercalation of imidazolium ionic liquids in kaolinites, Journal of Materials Chemistry, 17, 1476-1484.

Luo J.-J. and Daniel I. M. (2003). Characterization and modeling of mechanical behavior of polymer/clay nanocomposites. Composites Science and Technology, 63, 1607-1616

Murray H. H. (1988). Kaolin Minerals: their genesis and occurrences. In Hydrous Phyllosilicates, pp 67-89, ed. S.W. Bailey, Mineralogical Society of America.

Murray H. H. (2000). Traditional and new applications of kaolin, smectite, and palygorskite: a general overview. Applied clay Science, 17, 207-221.

Mbey J. A., Hoppe S. and Thomas F. (2012). Cassava-starch kaolinite composite film. Effect of clay content and clay modification on film properties. Carbohydrate polymers, 88(1), 213-222.

Njoya A., Nkoumbou C., Grosbois C., Njopwouo D., Njoya D., Courtin-Nomade A., Yvon J., and Martin F. (2006). Genesis of Mayouom kaolin deposit (western Cameroon). Applied Clay Science, 32, 125-140.

Njoya D., Hajjaji M., Baçaoui A. and Njopwouo D. (2010). Microstructural characterization and influence of manufacturing parameters on technological properties of vitreous ceramic materials. Materials characterization, 61, 289-295.

386 Njiomou Djangang C., Kamseu E., Kor Ndikontar M., Lecomte Nana G. L., Soro J., Melo U. 387 C., Elimbi A., Blanchart P. and Njopwouo D. (2011). Sintering behaviour of porous ceramic 388 kaolin-corundum composites: Phase evolution and densification. Materials Science and 389 Engineering $A, 528,8311-8318$. 
390 Nkoumbou C., Njoya A., Njoya D., Grosbois C., Njopwouo D., Yvon J. and Martin F. (2009).

391 Kaolin from Mayouom (Western Cameroon): Industrial suitability evaluation. Applied Clay $392 \quad$ Science, $43,118-124$.

393 Olejnik V. S., Posner A. M. and Quirk J. P. (1970). The intercalation of polar organic 394 compound into kaolinite. Clay Minerals, 8, 421-434.

395 Olejnik V. S., Aylmore L. A. G., Posner A. M. and Quirk J. P. (1968). Infrared Spectra of 396 Kaolin Mineral-Dimethyl Sulfoxide Complexes. The Journal of Physical Chemistry 72 (1), $397 \quad 241-249$.

398 Pavlidou S. and Papaspyrides C. D (2008). A review on polymer-layered silicate 399 nanocomposites. Progress in Polymer science, 33, 1119-1198.

400 Ray S. S. and Bousmina M. (2005). Biodegradable polymers and their layered silicate 401 nanocomposites: In greening the $21^{\text {st }}$ century materials world. Progress in Materials $402 \quad$ Science 50, 962-1079

403 Sugahara Y., Satokawa S., Kuroda K., And Kato C. (1988). Evidence for the formation of 404 interlayer Polyacrylonitrile in kaolinite. Clays and Clay Minerals, 36( 4), 343-348.

405 Wang B. X. and Zhao X. P. (2006). The influence of intercalation rate and degree of 406 substitution on the electrorheological activity of a novel ternary intercalated nanocomposite. 407 Journal of Solid State Chemistry, 179, 949-954.

408 Wilhelm H. M., Sierakowski M. R., Souza G. P., and Wypych, F. (2003). The influenced of 409 layered compounds on the properties of starch/layered compounds composites. Polymer $410 \quad$ International, 52, 1035-1044. 

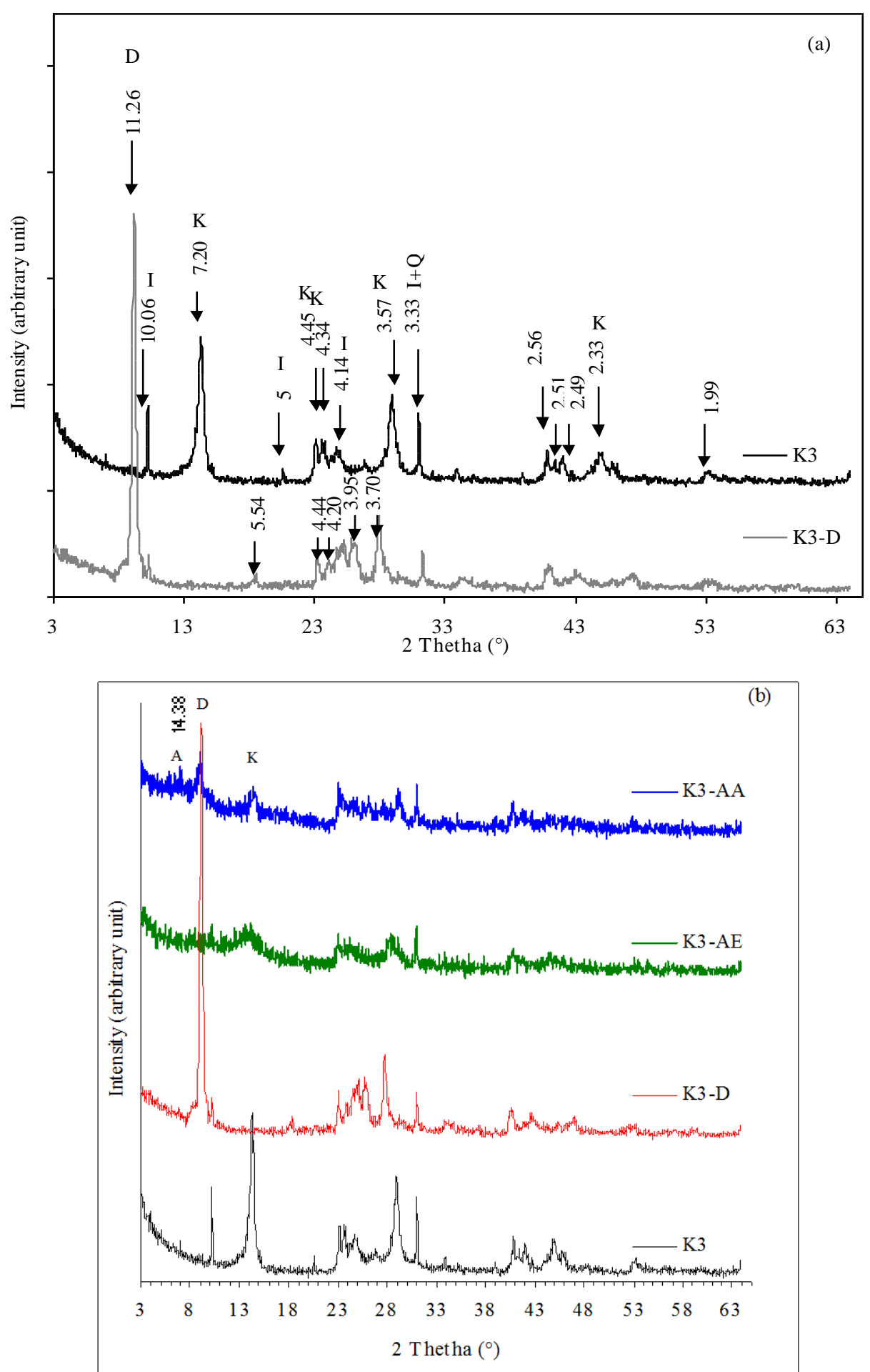

Figure 1: X-ray patterns of (a) the raw kaolinite and the DMSO-intercalated kaolinite (b) products of MDSO displacement using ammonium acetate (K3-AA) and ethyl acetate (K3$\mathrm{AE})$. 

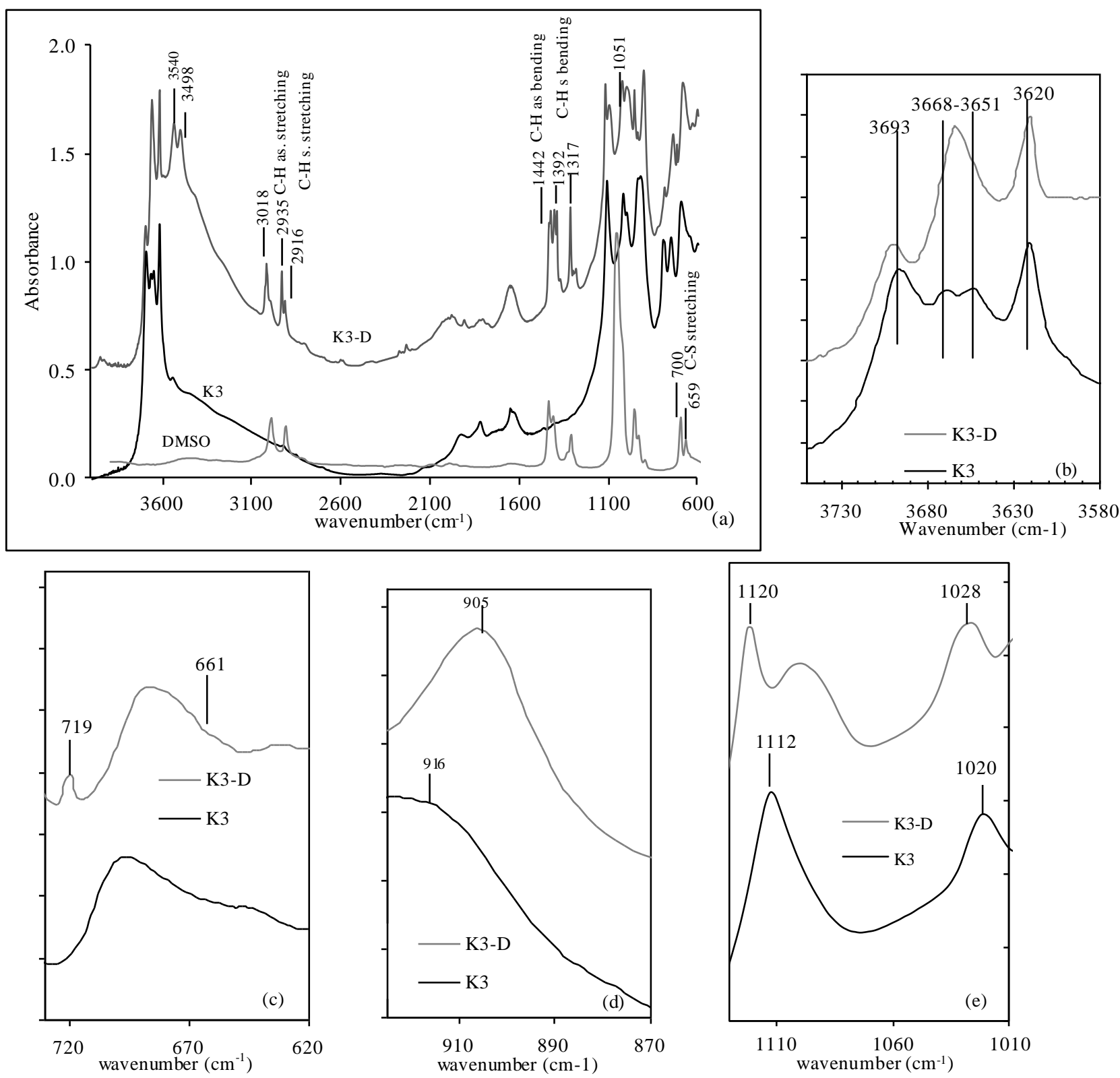

Figure 2: FTIR spectra: (a) complete spectra of DMSO-intercalated (K3-D), pure DMSO, and raw kaolinite $(\mathrm{K} 3)(b)$ kaolinite $\mathrm{OH}$ stretching band zone (c) stretching of $C-S$-C bonds of the DMSO molecule $(d)$ bending of the inner surface Al-OH of kaolinite (e) In plane vibrations of $\mathrm{Si}-\mathrm{O}$ 

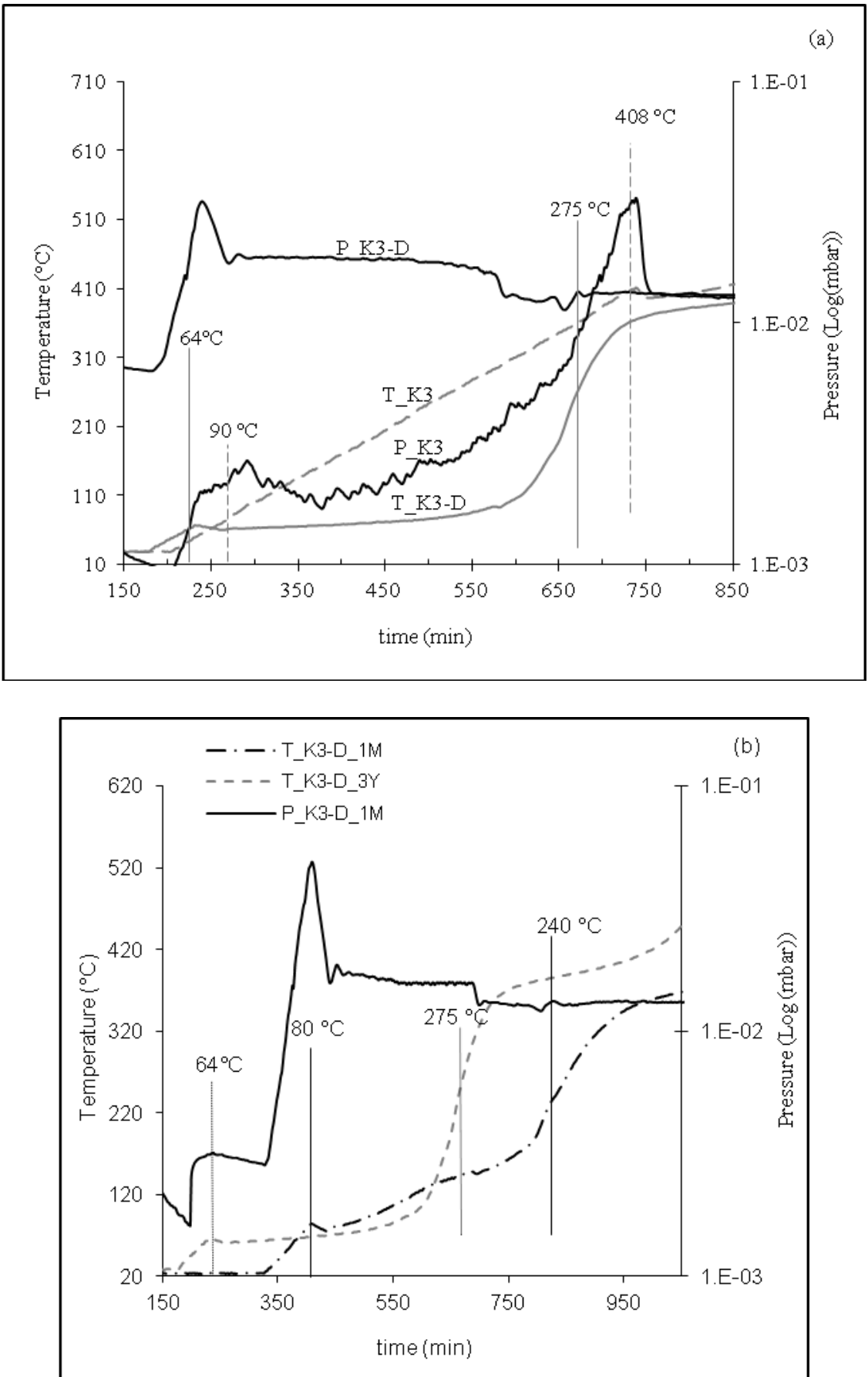

Figure 3: (a) Pressure and temperature evolution during CRTA of the DMSO intercalated 437 (K3-D) and the raw kaolinite (K3) (b) Comparison between a 3 year age (K3-D_3Y) and 1 month age (K3-D_1M) DMSO-Kaolinite. 


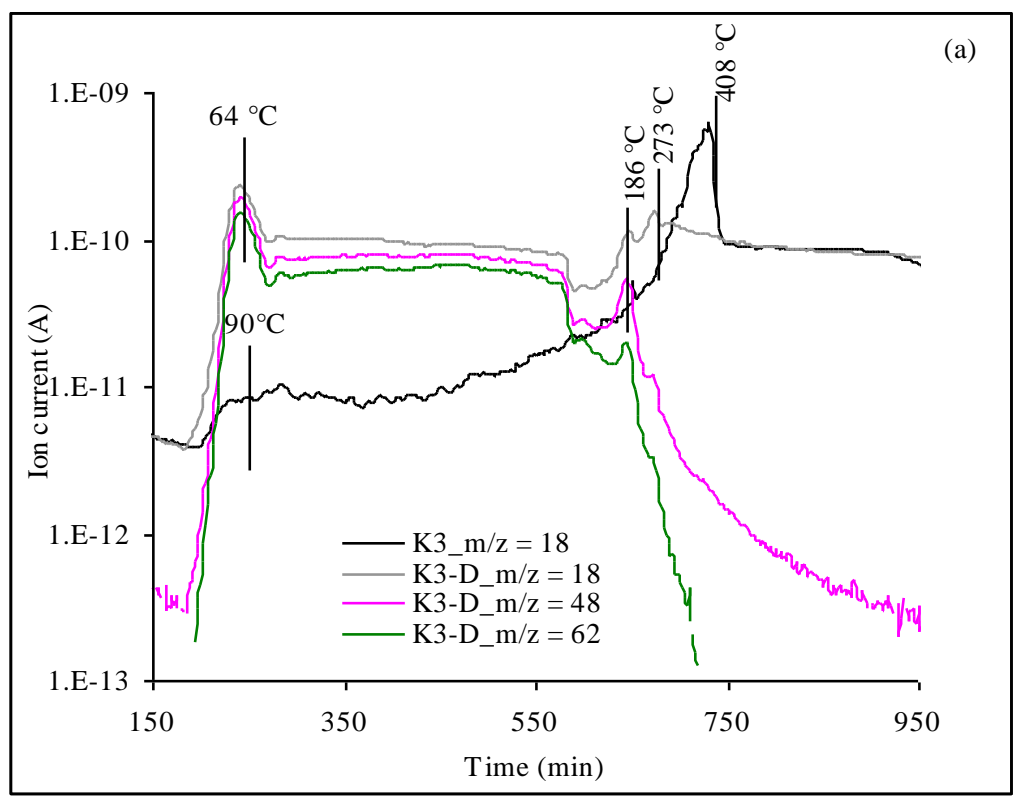

442

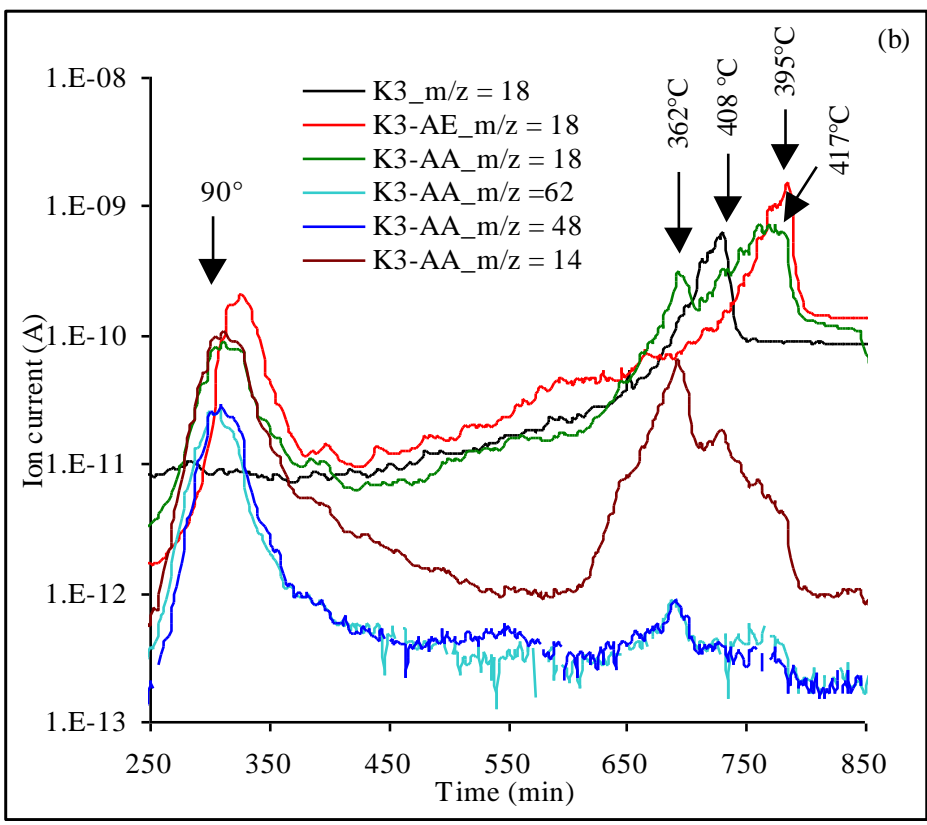




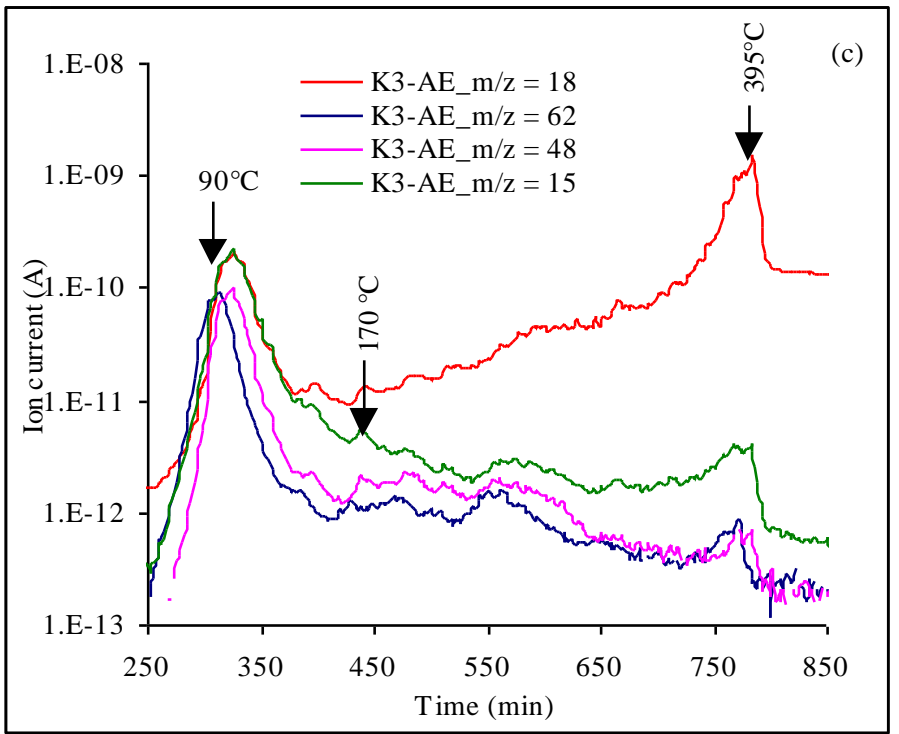

446 figure 4: Ion current due to water $(\mathrm{m} / \mathrm{e}=18), \mathrm{S}=\mathrm{O}(\mathrm{m} / \mathrm{e}=48)$ and $\mathrm{CH}_{3}-\mathrm{S}_{-}-\mathrm{CH}_{3}(\mathrm{~m} / \mathrm{e}=62)$

447 fragments during thermal treatment of (a) the DMSO-intercalated (K3-D) and the raw

448 kaolinite (K3); (b) products of DMSO displacement using ammonium acetate (K3-AA) and 449 ethyl acetate (K3-AE); (c) Trace DMSO evidence in K3-AE 
457
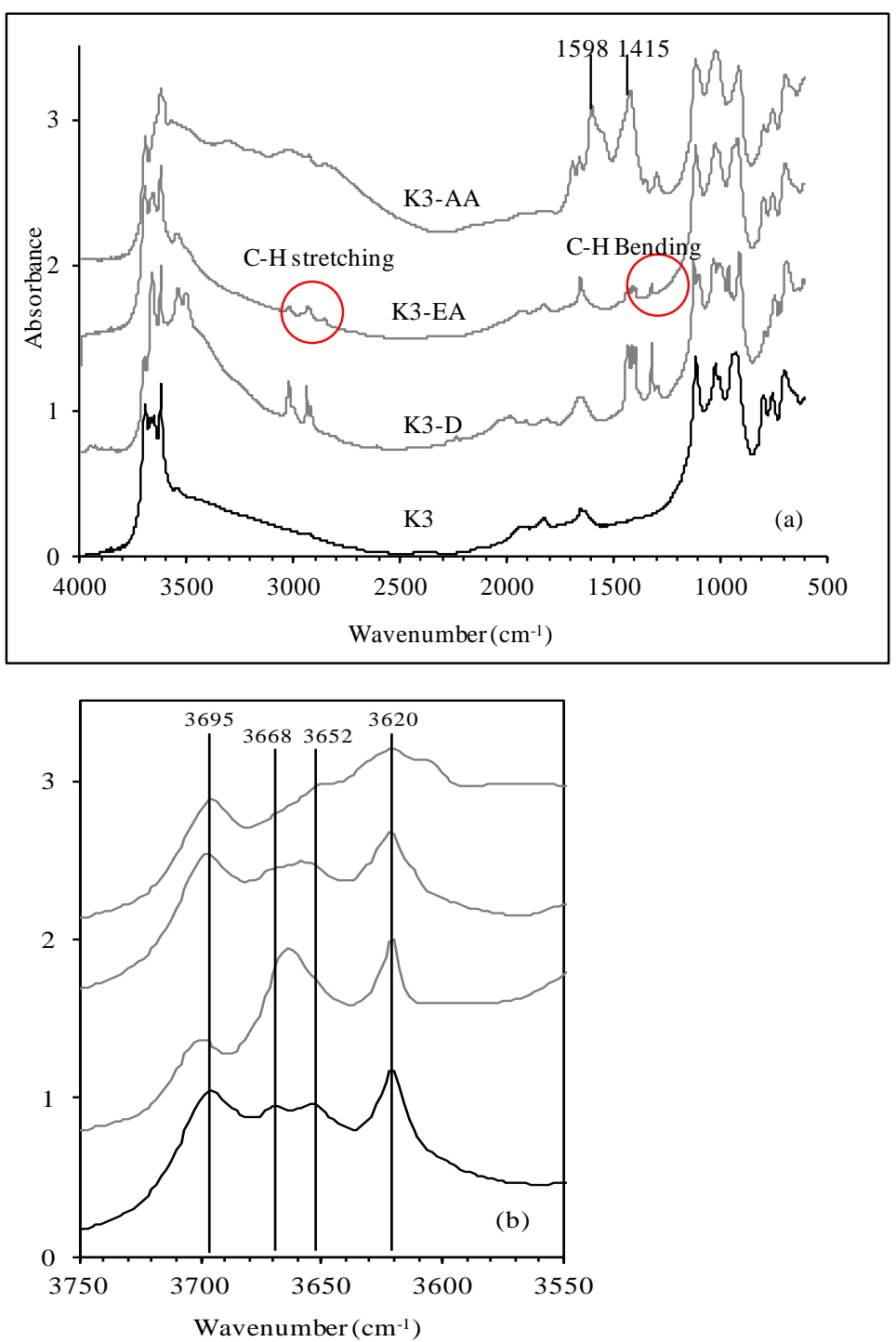

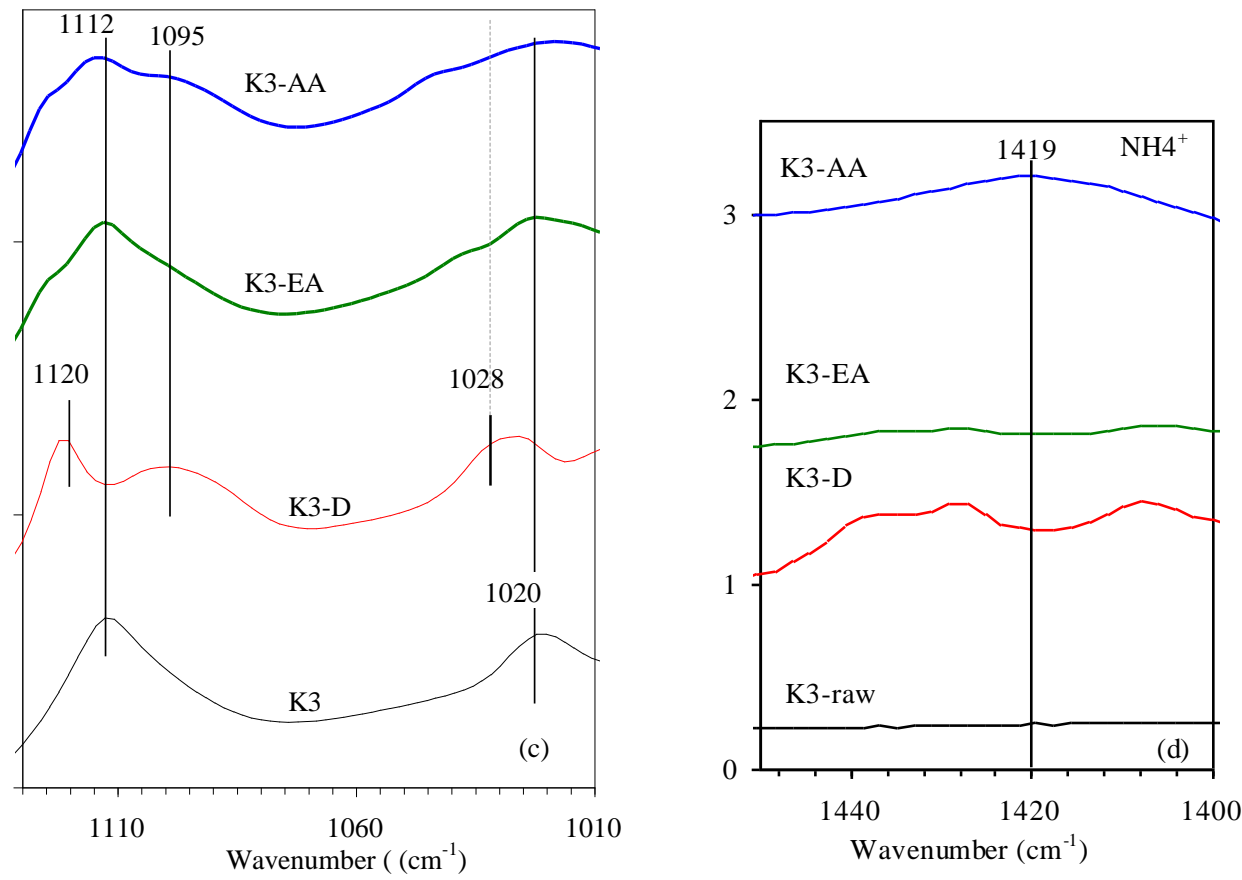

461
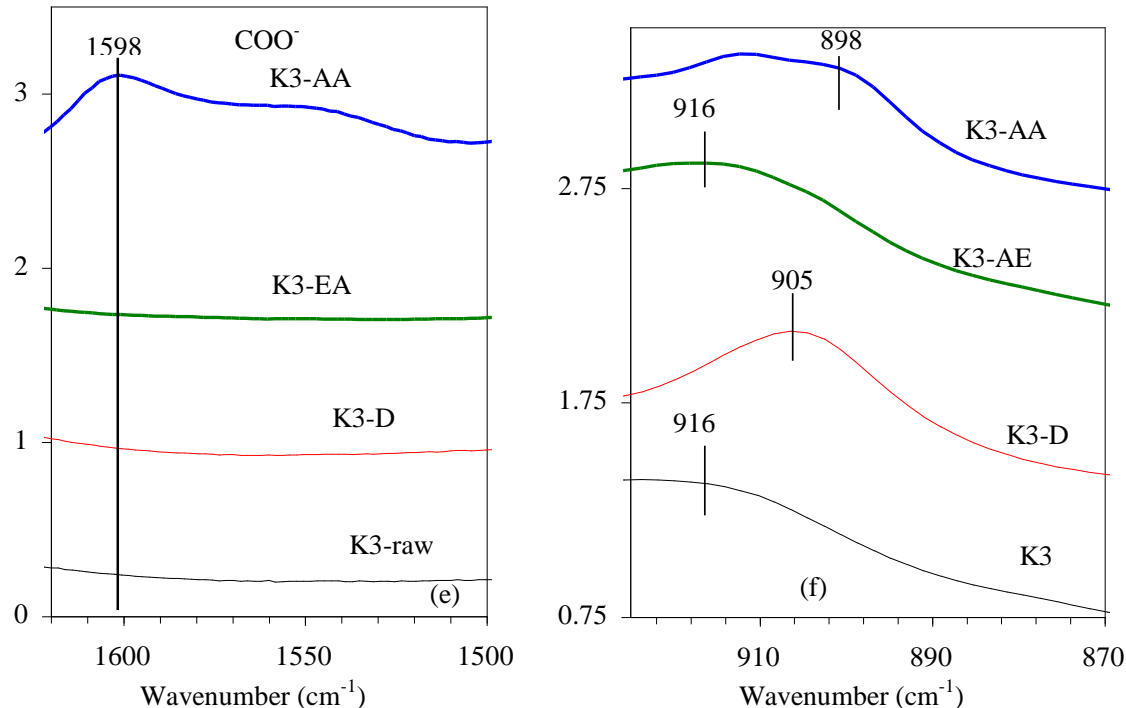

Figure 5: FT-IR spectra of the DMSO displacement in K3 kaolinite using ammonium acetate (K3-D-AA) and ethyl acetate (K3-D-EA): (a) complete spectra (b) O-H stretching domain (c) in plane vibrations modes of $\mathrm{Si}-\mathrm{O}\left(1112\right.$ and $\left.1020 \mathrm{~cm}^{-1}\right)$ and $\mathrm{S}=\mathrm{O}$ stretching $\left(1099 \mathrm{~cm}^{-1}\right)$. (d) 466 Evidence of $\mathrm{NH}^{+}$presence (e) Evidence of carbonyl vibration due to $\mathrm{COO}^{-}$group (f) Inner surface Al-OH bending modes. 


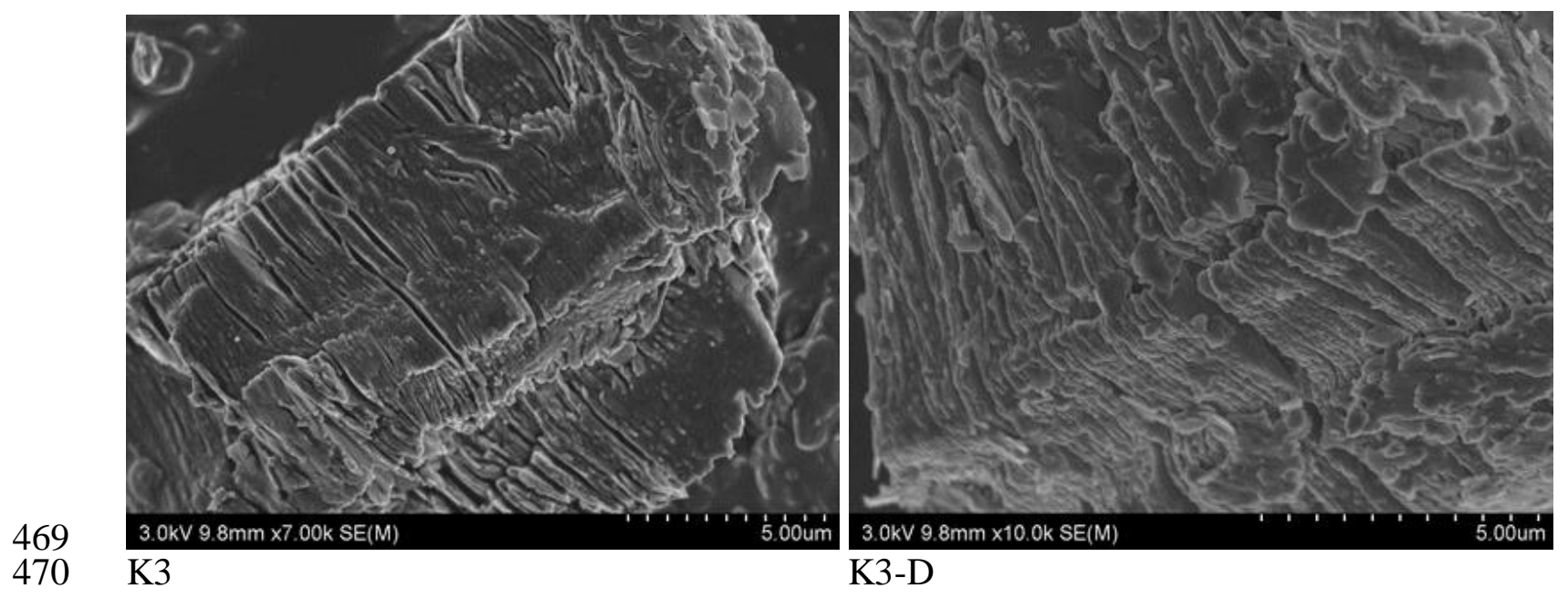

471 Figure 6: SEM micrographs of raw (K3) and DMSO-intercalated (K3-D) kaolinite.

472 